\title{
Vertical and lateral drift corrections of scanning probe microscopy images
}

\author{
P. Rahe, ${ }^{\text {a),b) }}$ R. Bechstein, ${ }^{\text {c) }}$ and A. Kühnle ${ }^{a)}$ \\ Fachbereich Physik, Universität Osnabrück, Barbarastraße 7, 49076 Osnabrück, Germany
}

(Received 28 October 2009; accepted 16 February 2010; published 20 May 2010)

\begin{abstract}
A procedure is presented for image correction of scanning probe microscopy data that is distorted by linear thermal drift. The procedure is based on common ideas for drift correction, which the authors combine to a comprehensive step-by-step description of how to measure drift velocities in all three dimensions and how to correct the images using these velocities. The presented method does not require any knowledge about size or shape of the imaged structures. Thus, it is applicable to any type of scanning probe microscopy image, including images lacking periodic structures. Besides providing a simple, ready-to-use description of lateral and vertical drift correction, they derive all formulas needed from the model of linear drift. (c) 2010 American Vacuum Society.

[DOI: 10.1116/1.3360909]
\end{abstract}

\section{INTRODUCTION}

Scanning probe microscopy (SPM) techniques, such as scanning tunneling microscopy (STM) or atomic force microscopy (AFM), have been proven to constitute extremely versatile tools for surface characterization that became indispensable in many areas of fundamental and applied research. ${ }^{1,2}$ However, SPM images are often found to be distorted by thermal drift or piezocreep, turning the determination of distances and angles into a challenging task. While piezocreep is a highly nonlinear effect that may be minimized by choosing adequate scanning parameters, thermal drift is often a linear effect that can be compensated or corrected. Thermal drift usually originates from differences in the coefficient of thermal expansion (CTE) and in the temperature diffusivity of the different materials in a SPM device. The scanner unit usually consists of piezoceramics while the sample holder and the housing is mainly made of metals. The sample can be any material. The following estimation is intended to emphasize the importance of thermal drift in SPM experiments. Metals have a typical CTE in the order of $10^{-5} \mathrm{~K}^{-1}$ and the typical dimensions in an SPM device are of the order of $10^{-2} \mathrm{~m}$. Assuming a change in temperature of only $1 \mathrm{~K} / \mathrm{h}$, a difference in the CTE of $10 \%$ between two materials already results in thermal drift of the order of $10 \mathrm{~nm} / \mathrm{h}$. Consequently, compensating thermal drift is usually required when SPM images are intended to be used for precise determination of both, distances and angles.

Here, we present a method for drift determination and image correction of SPM data to be applied after acquisition. We provide a straightforward step-by-step description of how to measure the drift velocities in all three dimensions and how to correct the images using these velocities. Most importantly, this method does not require any knowledge

\footnotetext{
${ }^{a}$ Present address: Institut für Physikalische Chemie, Fachbereich Chemie, Johannes Gutenberg-Universität Mainz, Jakob-Welder-Weg 11, 55099 Mainz, Germany.

${ }^{b)}$ Electronic mail: rahe@uni-mainz.de

${ }^{c}$ Present address: Interdisciplinary Nanoscience Center (iNano) and Department of Physics and Astronomy, University of Aarhus, Ny Munkegade, DK-8000 Århus C, Denmark.
}

about size or shape of the imaged structures. Thus, it is applicable to any type of SPM images, including those lacking periodic structures at all. We present all correction equations using the open-source SPM data visualization and analysis tool GWYDDION. ${ }^{3}$ All formulas used in this article are derived in detail, thus, the presented formulas are readily adaptable to other SPM data processing programs as well. Hence, with this recipe we analyze and combine several known strategies. The formalism presented here is based on linear drift only, however, the procedure can be extended to nonlinear drift in a straightforward manner.

The outline of the article is as follows. In Sec. II we will start by introducing existing methods for drift correction from literature. Special focus will be on applicability and limitations of these methods. Thereafter, we will explain our approach in a step-by-step fashion, starting with several important definitions (Sec. III) followed by the description of how to correct drift in vertical (Sec. IV) and lateral (Sec. V) direction. Every step will be deduced from simple geometry and will be illustrated by a practical example. Finally, we will discuss the applicability and main advantages of the described method (Sec. VI).

\section{EXISTING METHODS}

This section is intended to provide an overview over existing strategies for drift compensation and their limitations. A frequently used approach to reducing thermal drift is placing both scanner and sample in a cryostat. However, many application-oriented investigations involving chemical reactions or diffusion processes require room temperature or even elevated temperatures. Another possibility is to scan fast enough to make thermal drift become negligible. Videorate imaging experiments with atomic resolution, however, have been successfully demonstrated by only few STM groups, ${ }^{4-6}$ and atomic-resolution AFM experiments are still limited to a typical scan speed of less than 1 image/min. Thus, thermal drift is a very common problem in SPM.

Drift correction can be done either while measuring or after data acquisition has been completed. A very elegant experimental approach to compensate for drift during AFM 


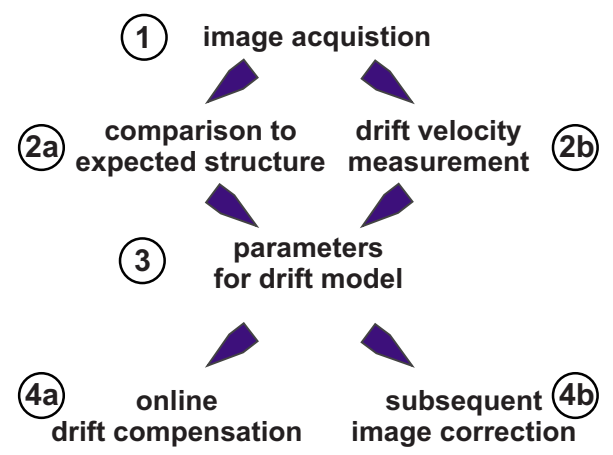

Fig. 1. (Color online) Schematic representation for drift correction methods. The main differences in drift correction methods presented so far occur during steps (2) and (4).

measurements was recently presented by Abe et al..$^{7-9}$ There, an atom-tracking procedure is used for determining the actual drift velocity, which is then fed into a feed-forward routine. For STM, this atom-tracking technique was introduced by Pohl and Möller ${ }^{10}$ and was analyzed in detail by Rerkkumsup et al. ${ }^{11}$ Atom tracking has been employed for studying the diffusion of adsorbates with STM (Refs. 12 and 13) and the identification and manipulation of single atoms with AFM (Refs. 14 and 15). Obviously, this kind of approach corrects the image based on drift parameters obtained from preceding data. For correcting SPM images after acquisition, several strategies are discussed in literature. All drift compensation and correction strategies split into methods based on (i) comparing properties such as periodicity or shape in the measured image to the expected surface structure and (ii) methods measuring drift velocities e.g., by tracking stationary features (see Fig. 1). All procedures provide parameters that are used to compensate the drift during measurement or correct recorded images to allow for exact length and angle measurements.

Correcting for drift has been widely discussed in literature ${ }^{7-9,16-25}$ and a large number of strategies have been presented. All strategies follow the paths sketched in Fig. 1 and can, thus, be classified by their strategy to derive the parameters for drift compensation [step (2)], by their drift model [step (3)] as well as by their drift compensation and correction procedure [step (4)]. The following overview highlights important methods published in recent years using Fig. 1 for classification.

Step (2a). Imaging known structures provides knowledge about the surface structure periodicity and shape. Then, drift correction parameters can be calculated from a comparison of the measured with the expected dimensions, angles, and shapes. For a hexagonal lattice this was described by Jørgensen $e t a l .{ }^{16}$ using paired lattice vectors from the reciprocal unit cell in Fourier space. For structures of circular shape Henriksen and Stipp ${ }^{17}$ presented a method that allows for calculating drift parameters from the transformation of the imaged ellipse back to a circle. Staub et al. ${ }^{18}$ showed how to deduce drift parameters from correct calibration factors.
Drift correction along the fast scan direction can only be done by shifting the individual lines along the fast scan direction. A straight step structure can, for example, serve as linear structure. This strategy is implemented in GWYDDION. ${ }^{26}$ For determining lateral drift in two directions, the scan direction needs to be rotated. The basis of all these approaches constitutes a severe limitation: The surface structure must be known.

Step (2b). In absence of knowledge about the imaged surface structure, a minimum of two images is required for drift correction. For images of the same scanning direction autocorrelation is the standard technique. ${ }^{19,20}$ Drift velocities in lateral directions are derived from the resulting autocorrelation image by dividing the separation of the two major peaks by the elapsed time. ${ }^{19,20}$ The prerequisite for this approach is a significant corrugation in the two images to achieve a reliable autocorrelation spectrum.

Images of different scanning directions can be corrected by a strategy that was described by Yurov and Klimov. ${ }^{21}$ Corresponding formulas can be found in Refs. 22 and 23. A drawback of this strategy is that piezocreep may occur and therefore two sequential images with different scanning directions might be shifted relative to each other, resulting in incorrect drift velocities. ${ }^{27}$

Woodward et al. ${ }^{24}$ showed how to derive drift velocities from calculating the average lattice vectors of two images, scanned in opposite directions. The average constitutes a first-order approximation of the correct lengths. However, this approach is, again, only applicable if the images show periodic structures.

To improve efficiency and speed, complex algorithms based on image analysis are used to find the relative alignment of consecutive images. A survey of these image registration techniques is available in Ref. 28. A subpixel registration $^{29}$ is implemented in the autoalign tool of GXSM. ${ }^{30}$ Some of these strategies are optimized for a specific sample system and may not be applicable to every SPM image.

In most scanning probe controllers, drift velocities are determined by cross correlation of two images or by tracking a single feature. The results are used for an online drift correction.

The strategies mentioned so far deal with drift correction in lateral directions. Drift along the vertical axis was also discussed by Yurov and Klimov ${ }^{21}$ by using one upscanned and one downscanned image and by Lapshin, ${ }^{22}$ where counterscanned images are used to calculate coefficients for a linear transformation.

Step (3). Most drift models are based on modeling a linear transformation between the coordinates of the original sample system to the distorted scanner system. This linear transformation involves scaling $\hat{\mathbf{S}}$ along both axes, as well as shearing $\hat{\mathbf{H}}$ along the fast and compression/expansion $\hat{\mathbf{C}}$ along the slow scanning direction. This transformation is often introduced as matrix operation ${ }^{17,18,24}$ 


$$
\hat{\mathbf{D}}=\hat{\mathbf{S}} \hat{\mathbf{H}} \hat{\mathbf{C}}
$$

with $\hat{\mathbf{D}}$ representing the total transformation operation. For correcting the images, the inverse transformation $\hat{\mathbf{D}}^{-1}$ has to be applied.

Besides this linear model, it has been suggested to predict the drift velocity after finishing the acquisition of the preceding image, ${ }^{25}$ which has been realized using a neural network.

Step (4a). Online drift compensation is performed by directly using the determined drift velocities for the subsequent data acquisition. For this feed-forward technique, timevarying voltage offsets are applied to the scanner tube additionally to the scan signals. Most scanning probe controllers can easily generate these signals.

Step (4b). For image correction, discrete pixels-each representing a physical value-need to be mapped to new (correct) positions. Due to the discretization, interpolation techniques need to be used and should critically be checked for their physical justification.

The strategy described in this article relies on tracking one stationary feature in at least two sequential images [step (2b)] of identical scan direction. Therefore, periodic structures or regular shaped features are not required as long as single features can be identified to be stationary. Furthermore, no information about the surface structure is needed. In particular, images at all scales can be corrected with this strategy. ${ }^{31}$ Most importantly, the drift compensation procedure can be verified to be exact by applying the method to image sets scanned in different directions, as will be shown in Sec. V.

\section{DEFINITIONS AND METHODS}

Usually, SPM images are obtained using forward and backward, up and down lateral scanning directions, as depicted in Fig. 2. Forward and backward, the fast scan directions, are indicated by blue horizontal arrows. The slow scan directions, up and down, are indicated by yellow vertical arrows. Note that for simplicity, the identical coordinate system [hereafter referred to as forward-down-coordinate (FDC) system] is used for all images. Thus, the first pixel of an image does not necessarily coincide with the origin of the coordinate system as indicated by the red crosses in Fig. 2.

A single image with the physical dimensions width $B$ and height $H$ consists of $N$ lines and $M$ pixels per line. Images are obtained with a scan speed given as raster time, e.g., $u=1 \mathrm{~ms} /$ pixel. The total acquisition time of an image is the time elapsed between first and last pixel of the image. In the most general case, where each line is scanned in forward and backward direction, the total time elapsed between first and last pixels of an image corresponds to the total acquisition time $T$ per pair of forward and backward image. It is essential to note that $T$ may differ from $2 \times N \times M \times u$ as delays in the scan and acquisition electronics often result in an additional offset. It is absolutely necessary to determine $T$ as precisely as possible.

The example SPM images shown in this article are noncontact AFM raw data images obtained at room temperature
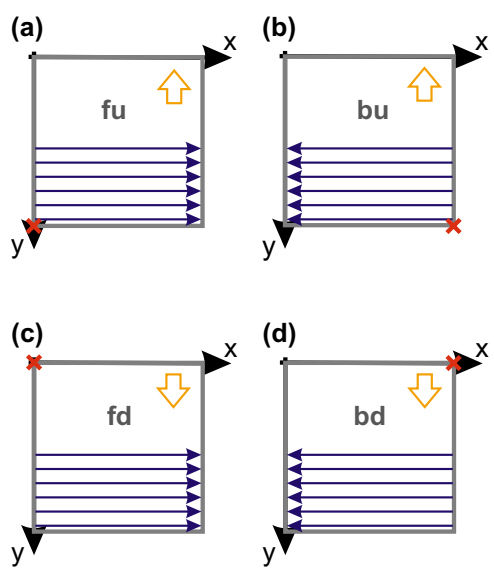

FIG. 2. (Color online) Illustration of the lateral scanning directions. The fast scanning directions, forward and backward, are indicated by blue horizontal arrows. Up and down, the slow scan directions, are marked by yellow vertical arrows. Thus, four types of images are typically recorded: forward up (a), backward up (b), forward down (c), and backward down (d). The up and down images are recorded pairwise. The red crosses indicate the first pixel in each image. All image types are processed in a FDC system with $(0,0)$ in the upper left corner.

on a rutile $\mathrm{TiO}_{2}(110)$ sample codoped with chromium and antimony. ${ }^{31}$ The images have a size of $N=M=500$ pixel and were acquired with a scan speed of $u=1 \mathrm{~ms} /$ pixel. The total acquisition time per pair of forward and backward images was $T=557 \mathrm{~s}$ for all images shown here.

When performing image correction for measuring distances and angles in a reliable manner, the calibration of the microscope needs to be carefully and frequently checked. During the experiments with our microscope, it became obvious that lateral dimensions have to be corrected by a factor of

$$
\begin{aligned}
& C_{x}=0.88 \pm 0.04, \\
& C_{y}=0.88 \pm 0.04,
\end{aligned}
$$

for both $x$ and $y$ directions. These factors were confirmed in experiments on $\mathrm{Si}(111)(7 \times 7), \quad \mathrm{TiO}_{2}(110), \quad$ and $\mathrm{CaCO}_{3}(10 \overline{1} 4)$. All raw data images need to be rescaled by these rescaling factors prior to the drift correction procedure. Note that our drift correction procedure is also applicable to noncalibrated data, but the scale of the corrected images as well as the determined drift velocities are incorrect by the same factors in this case. However, drift correction without the knowledge of correct calibration factors is nevertheless possible and in fact necessary for the initial determination of precise calibration factors.

\section{VERTICAL DRIFT}

After rescaling it is necessary to correct topography images for vertical drift. In the most simple case, an untilted sample is imaged. To measure the vertical drift velocity, height profiles along the slow scan direction ( $y$ direction) taken from consecutive up and down images are plotted, as 


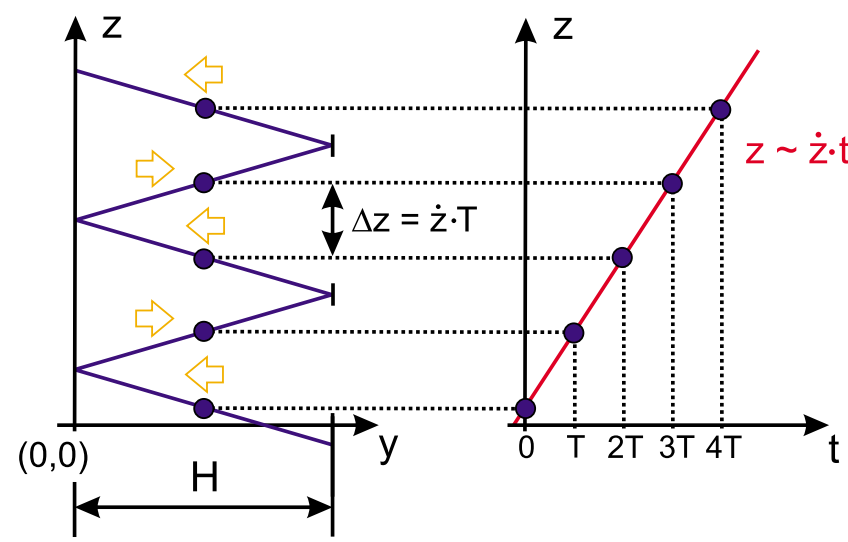

FIG. 3. (Color online) Vertical drift correction scheme in case of an untilted sample. Line plots along the slow scan direction (image height $H$ ) taken from consecutive up and down images are depicted to the left. The average values are indicated by blue circles. The height difference between these values correspond to $\dot{z} \cdot T$. A plot of the average values vs acquisition time reveals a curve with constant slope $\dot{z}$.

depicted by the blue solid lines in Fig. 3. In case of a positive (negative) vertical drift velocity, these curves exhibit a monotonic increase (decrease) in $z$.

A constant difference in height $\Delta z=\dot{z} \cdot T$ between consecutive images can be measured if the drift velocity is constant. For simplicity, we consider the average values from each complete image, which are plotted versus the acquisition time $T$ as indicated by blue circles in Fig. 3. This procedure, furthermore, allows to use images of different sizes and reduces the influence of image corrugation as well as statistical outliers. The average values can be used as long as the typical height of features in the image is much smaller than the lateral dimensions of the image and as long as the vertical drift velocity is not much smaller then the lateral drift velocities. The vertical drift velocity $\dot{z}$ can now be derived from a linear least-squares fit to the average values. The slope of the resulting curve equals $\dot{z}$.

Usually, the surface normal vector is not parallel to the scanner tube. The crystal often has a miscut in the order of $0.1^{\circ}$. Furthermore, misalignment of the AFM cantilever or STM tip is virtually unavoidable. Most importantly, the sample surface itself might not be planar per default. Surfaces can consist of vicinal facets or of terraces of different crystallographic faces.

These variations result in a tilted sample surface, which has to be compensated by the distance feedback loop. A surface tilt by an angle $\beta$ results in an additional height difference of $\pm H \tan \beta$ along the slow scan direction of an up or down image of lateral size $H$, respectively. This situation is depicted in Fig. 4. The total height differences in up and down images along $y$ are

$$
\begin{gathered}
\Delta z_{\uparrow}=\dot{z} \cdot T+H \tan \beta, \\
\Delta z_{\downarrow}=\dot{z} \cdot T-H \tan \beta .
\end{gathered}
$$

However, the height difference between the average values of consecutive up and down images is

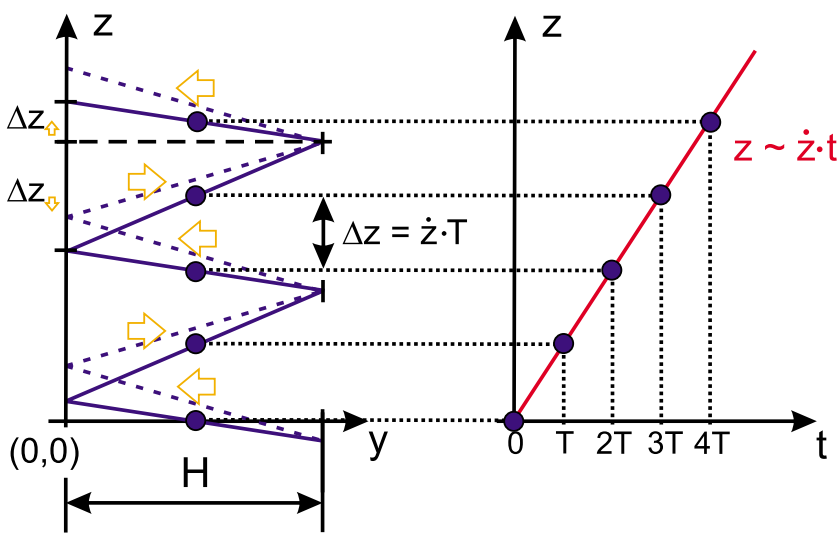

FIG. 4. (Color online) Vertical drift correction scheme in case of a tilted sample. In analogy to Fig. 3, line plots along the slow scan direction are depicted to the left. The average values are indicated by blue circles. The height difference between average values from consecutive up and down images still corresponds to $\dot{z} \cdot T$. Thus, the plot of the average values vs acquisition time reveals a constant slope of $\dot{z}$, independent of the sample tilt.

$$
\Delta z=\frac{1}{2}\left(\Delta z_{\uparrow}+\Delta z_{\downarrow}\right)=\dot{z} \cdot T .
$$

Hence, the already described recipe for obtaining the vertical drift velocity is also valid for tilted samples. Furthermore, sample tilt will not be corrected if the drift velocity is derived from the change in the average value of consecutive up and down topography images. Note that surface tilt can only be compensated by a rotation. This has to be differentiated from vertical drift, which is compensated by a projection. ${ }^{21}$

After obtaining a value for the vertical drift velocity, we now explain a procedure to correct a given image for vertical drift. A single topography image consists of $N$ lines with $M$ pixels/line. Thus, it can be written as a $(N \times M)$ matrix $Z$ of height values $z_{i j}$,

$$
Z=\left(z_{i j}\right)_{i=1, \ldots, N, j=1, \ldots, M} .
$$

For correcting the vertical drift of several images considering a correct relative alignment of all images with respect to each other, we derive one formula for each type of image. Accordingly, the following formulas have to be used for a forward $(f)$ or backward $(b)$ image in up $(u)$ or down $(d)$ direction, respectively,

$$
\begin{aligned}
& z_{i j}^{\mathrm{fu}}=z_{i j}^{\text {orig }}-\dot{z} \frac{T}{2 M N} \cdot[2 M(N-i)+j], \\
& z_{i j}^{\mathrm{bu}}=z_{i j}^{\text {orig }}-\dot{z} \frac{T}{2 M N} \cdot[2 M(N-i+1)-j+1], \\
& z_{i j}^{\mathrm{fd}}=z_{i j}^{\text {orig }}-\dot{z} \frac{T}{2 M N} \cdot[2 M(N+i-1)+j], \\
& z_{i j}^{\mathrm{bd}}=z_{i j}^{\text {orig }}-\dot{z} \frac{T}{2 M N} \cdot[2 M(N+i)-j+1] .
\end{aligned}
$$

All coordinates are given in the FDC system as introduced in Fig. 2. For a correct relative alignment of a set of consecutive up and down images, an offset of $-\dot{z} T$ has been included 


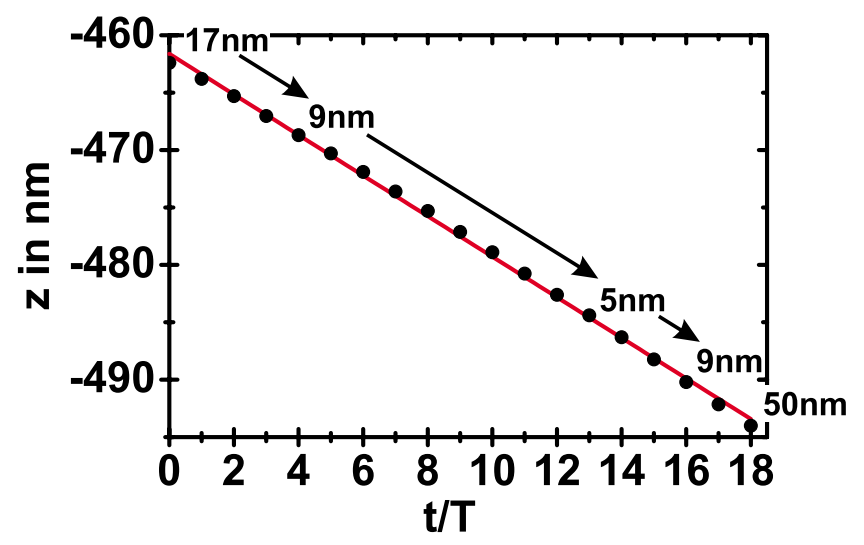

FIG. 5. (Color online) Average values of 19 consecutively obtained topography images. The image size was changed as indicated by the values in the graph. The linear least-squares fit to the data reveals a slope of $\dot{z}$ $=-(3.15 \pm 0.03) \mathrm{pm} / \mathrm{s}$. The total acquisition time of this image series was $\sim 3 \mathrm{~h}$.

in the formulas for the down images [Eqs. (10) and (11)]. An offset of $-2 \dot{z} T$ needs to be added to each following set of up and down images if the exact relative alignment is important.

In Fig. 5 an example is given, showing the average values of 19 consecutively obtained topography images. The image size is indicated in the graph. From a least-squares fit to the data the drift velocity is determined to be $\dot{z}$ $=-(3.15 \pm 0.03) \mathrm{pm} / \mathrm{s}$. The assumption of linear drift is obviously fulfilled.

For the image correction, a simple algorithm can now be used, reflecting Eqs. (8)-(11). Here, we present the pseudocode for a single image,

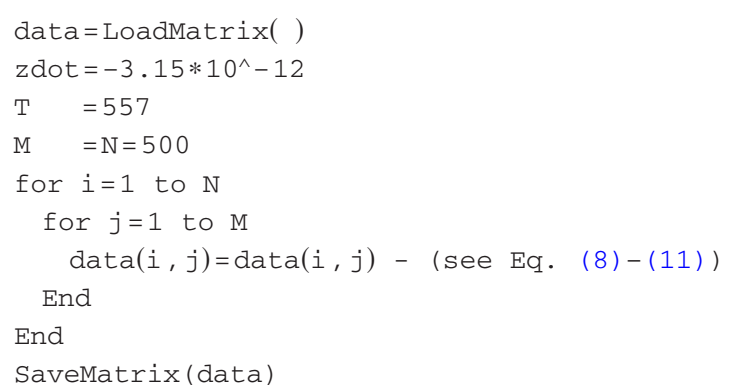

Applying this algorithm results in images corrected for linear vertical drift. In case of nonlinear drift a simple approach would be to fit the $z(t)$ curve with a polynomial function that can often be approximated by the first-order Taylor expansion for every image.

\section{LATERAL DRIFT}

Lateral drift is corrected by tracking a stationary feature in consecutively obtained images. This easy but robust principle is often used in scanning probe controllers. It can be performed on any type of lateral scan image. Thus, the recipe presented here is not limited to images revealing periodic structures of known size and shape. More sophisticated strat- (a)

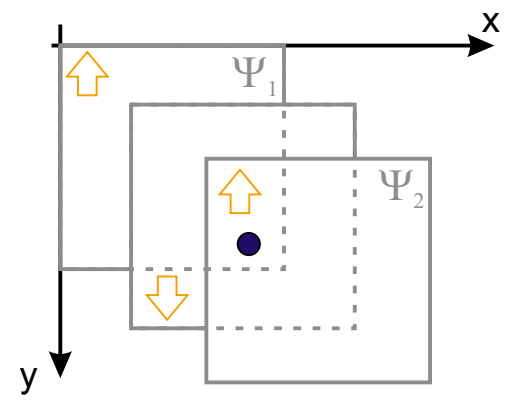

(b)

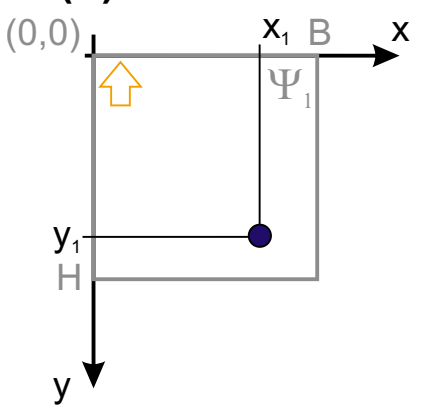

(c)

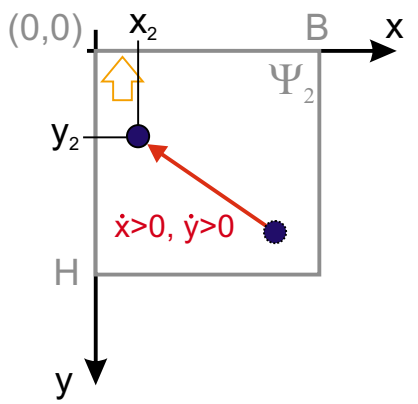

FIG. 6. (Color online) Lateral drift velocity values can be obtained by tracking a stationary feature in consecutive up (or down) images. Here, a stationary feature (blue circle) is identified in consecutive up images $\Psi_{1}$ and $\Psi_{2}$. The image frame moves to the lower right in this example (a). Accordingly, the feature appears to move to the upper left [(b) and (c)]. We define the corresponding drift velocity values to be positive.

egies as discussed in Sec. II, step (2b) may be used here to determine the lateral drift velocities from the relative alignment of the images automatically. Note that all images are processed in a FDC system.

For simplicity, we consider only linear drift with constant drift velocity components $\dot{x}$ and $\dot{y}$. We define the drift velocity values to be positive if consecutively obtained images move in the $x$ and $y$ directions, as depicted in Fig. 6(a). Accordingly, stationary features appear to move to the upper left in this case, as indicated in Figs. 6(b) and 6(c). The position of a stationary feature should be measured either in consecutive up or in consecutive down images to minimize the influence of piezocreep. Then, the drift velocities $\dot{x}$ and $\dot{y}$ are obtained from the positions by

$$
\begin{aligned}
& \dot{x}=\frac{x_{1}-x_{2}}{\Delta t}, \\
& \dot{y}=\frac{y_{1}-y_{2}}{\Delta t} .
\end{aligned}
$$

The time $\Delta t$ elapsed between the appearance of the feature in images $\Psi_{1}$ and $\Psi_{2}$ is calculated according to

$$
\Delta t_{\uparrow}=2 T+\left(y_{1}-y_{2}\right) \frac{T}{H},
$$


(a)

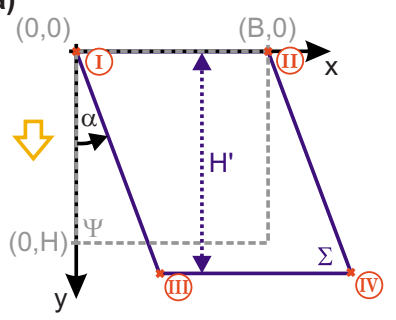

(b)

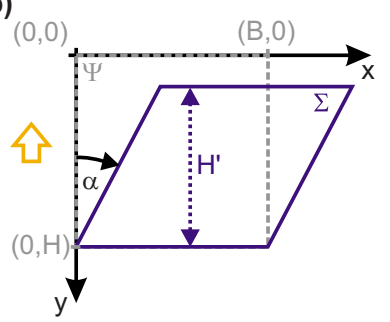

FIG. 7. (Color online) Scheme indicating the changes in a down (a) and an up (b) image due to lateral drift correction. The acquired image $(\Psi)$ is indicated by a rectangular dashed gray box of width $B$ and height $H$. The image corrected for lateral drift $(\Sigma)$ is indicated by a solid blue parallelogram. The effects of positive drift velocities $\dot{x}$ and $\dot{y}$ are shown. The images are sheared by an angle $\alpha$ due to drift in the $x$ direction. Drift in the $y$ direction is represented by the height difference $H-H^{\prime}$. The width of the corrected image is still $B$ and the scan lines are still parallel to the $x$ direction after drift correction, as compression in $x$ direction and shearing of each scan line is negligible.

$$
\Delta t_{\downarrow}=2 T-\left(y_{1}-y_{2}\right) \frac{T}{H},
$$

depending on whether the positions of the stationary feature were obtained from two consecutive up $(\uparrow)$ or down $(\downarrow)$ images. Note that the drift velocities obtained from up as well as from down images are identical. Hence, at least two up or two down images are required. However, for a reliable drift correction as much images as possible should be used.

For the drift correction procedure, we first define two systems: The system $\Psi$ as already introduced in Fig. 6 represents the image as acquired in the experiment. The system $\Sigma$ represents the image corrected for lateral drift. Positions in $\Psi$ and in $\Sigma$ are measured in the same FDC system, as introduced in Fig. 2, and are indicated by indices $\left(\begin{array}{l}x_{\Psi} \\ y_{\Psi}\end{array}\right) \in \Psi$ and $\left(\begin{array}{l}x_{\Sigma} \\ y_{\Sigma}\end{array}\right) \in \Sigma$, respectively. The acquired image is rectangular with the width $B$ and the height $H$. The corrected image is a parallelogram. The distortion induced by linear drift correction can be described by two parameters, as introduced in Fig. 7: The angle $\alpha$, which shears the image due to drift in the $x$ direction and the difference $H-H^{\prime}$, representing a height compression or expansion due to drift in the $y$ direction. The width of the corrected image is still $B$ and the scan lines are still parallel to the $x$ direction. Compression in the $x$ direction and shearing of each single scan line is negligible, as the acquisition time for a single scan line is smaller by a factor of $2 N$ compared to the acquisition time of a whole image.

The parameters $\alpha$ and $H-H^{\prime}$ are related to the drift velocities by simple geometry according to

$$
\begin{aligned}
& \dot{x} T=H^{\prime} \tan \alpha, \\
& \dot{y} T=H-H^{\prime},
\end{aligned}
$$

for an up image, and

$$
\dot{x} T=H^{\prime} \tan \alpha,
$$

$$
\dot{y} T=H^{\prime}-H,
$$

for a down image. The orientation of the angle is depicted in Fig. 7. Note that the shearing angle $\alpha$ is different in up and down images.

The drift correction will be carried out by a linear distortion according to the ansatz

$$
\begin{aligned}
& x_{\Psi}=P_{x}\left(x_{\Sigma}, y_{\Sigma}\right)=a_{0}+a_{1} x_{\Sigma}+a_{2} y_{\Sigma}, \\
& x_{\Psi}=P_{y}\left(x_{\Sigma}, y_{\Sigma}\right)=b_{0}+b_{1} x_{\Sigma}+b_{2} y_{\Sigma} .
\end{aligned}
$$

The coefficients $a_{i}, b_{i}$ have to be calculated for up and down images separately.

To derive the coefficients $a_{i}, b_{i}$ in Eqs. (20) and (21) for a down image, we consider corner points I-III as marked in Fig. 7(a): point I,

$$
\begin{aligned}
& P_{x, \downarrow}(0,0)=a_{0}=0, \\
& P_{y, \downarrow}(0,0)=b_{0}=0,
\end{aligned}
$$

point II,

$$
\begin{aligned}
& P_{x, \downarrow}(B, 0)=a_{1} B=B \Rightarrow a_{1}=1, \\
& P_{y, \downarrow}(B, 0)=b_{1} B=0 \Rightarrow b_{1}=0,
\end{aligned}
$$

point III,

$$
\begin{aligned}
& P_{x, \downarrow}\left(H^{\prime} \tan \alpha, H^{\prime}\right)=H^{\prime} \tan \alpha+a_{2} H^{\prime}=0 \Rightarrow a_{2}=-\tan \alpha, \\
& P_{y, \downarrow}\left(H^{\prime} \tan \alpha, H^{\prime}\right)=b_{2} H^{\prime}=H \Rightarrow b_{2}=\frac{H}{H^{\prime}} .
\end{aligned}
$$

Using Eqs. (18) and (19), the final result for the polynoms for the down image are

$$
\begin{aligned}
& x_{\Psi}=P_{x, \downarrow}\left(x_{\Sigma}, y_{\Sigma}\right)=x_{\Sigma}-\frac{\dot{x} T}{H+\dot{y} T} y_{\Sigma}, \\
& y_{\Psi}=P_{y, \downarrow}\left(x_{\Sigma}, y_{\Sigma}\right)=\frac{H}{H+\dot{y} T} y_{\Sigma} .
\end{aligned}
$$

The coefficients for the correction of up images can be calculated in complete analogy. The resulting polynomial equations are

$$
\begin{aligned}
& x_{\Psi}=P_{x, \uparrow}\left(x_{\Sigma}, y_{\Sigma}\right)=\frac{-\dot{x} T H}{H-\dot{y} T}+x_{\Sigma}+\frac{\dot{x} T}{H-\dot{y} T} y_{\Sigma}, \\
& y_{\Psi}=P_{y, \uparrow}\left(x_{\Sigma}, y_{\Sigma}\right)=\frac{H}{H-\dot{y} T}\left(y_{\Sigma}-\dot{y} T\right) .
\end{aligned}
$$

To carry out the drift correction, the drift velocities $\dot{x}$ and $\dot{y}$ are determined by tracking several features in consecutive images. Features must be tracked in either consecutively obtained up or down images. Mixing up and down images might result in incorrect drift velocities. ${ }^{27}$ However, comparing the results from a set of up images and a set of down images represents an elegant procedure to verify the obtained drift velocities. To minimize the error in the drift measure- 


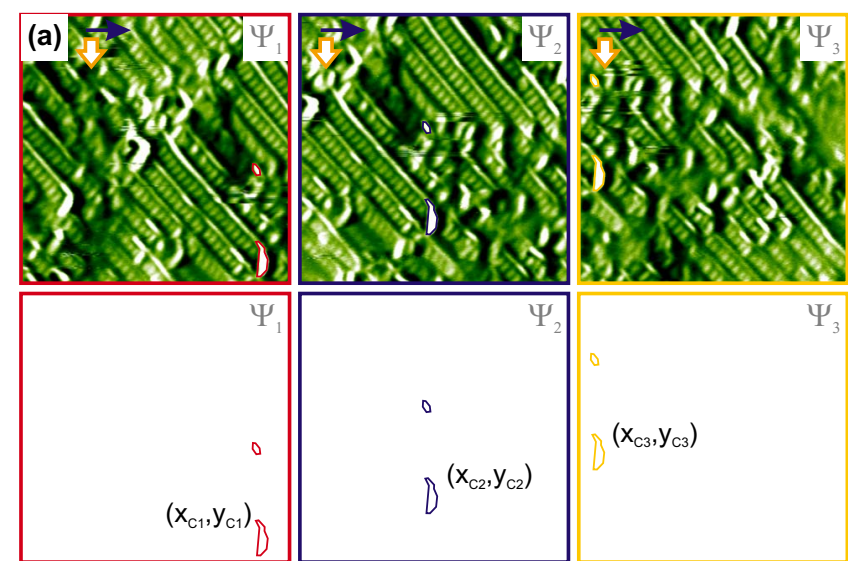

(b)
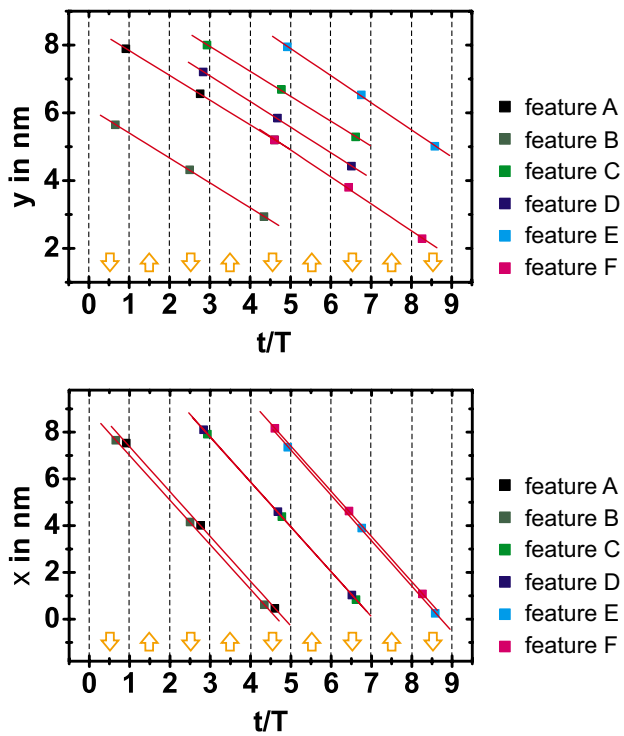

FIG. 8. (Color online) Tracking one feature (exemplary shown in feature C) in consecutive down images (a). The image size is $9.8 \mathrm{~nm}$ and the time per image is $557 \mathrm{~s}$. The coordinates $x(t)$ and $y(t)$ of six features in total, tracked in several images are plotted (b). Due to large drift, only three images overlap. The end of each image pair is indicated by vertical dashed lines. The drift velocities are obtained from least-squares fits to the data.

ment, as many features as possible should be tracked. Though, the magnitude of the drift limits the number of images showing the same individual feature.

Figure 8(a) shows three exemplary down images and two features marked in every image. Figure 8(b) represents $x(t)$ and $y(t)$ plots of several features tracked in five images in total. From least-squares fits to the $x(t)$ and $y(t)$ data, the drift velocities can be easily determined.

At this point, the assumption of linear drift can and should be checked. In the case of strong deviations from linear drift, a nonlinear model should be used and Eqs. (20) and (21) have to be modified accordingly.

By averaging over all tracked features of Fig. 8, we determine the lateral drift velocities in our example to be

$$
\dot{x}=(3.44 \pm 0.03) \mathrm{pm} / \mathrm{s},
$$

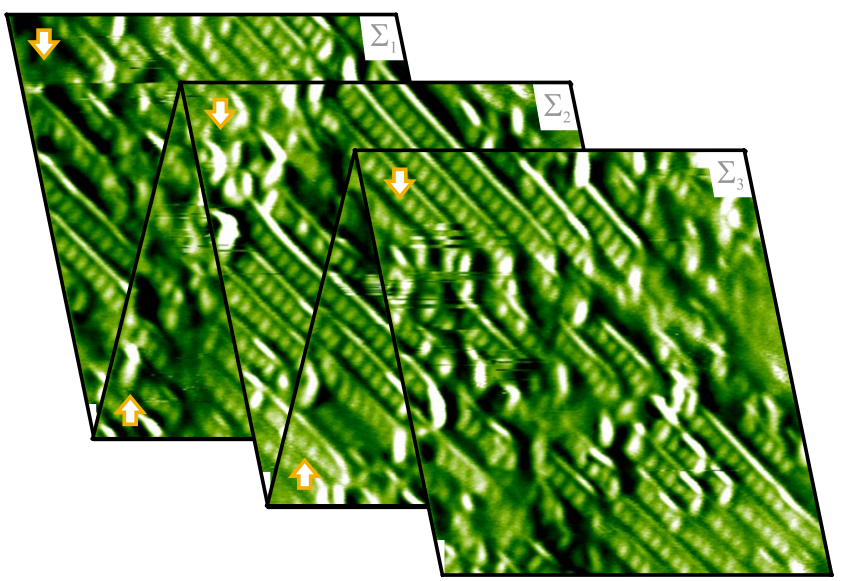

FIG. 9. (Color online) Merged images corrected for lateral drift. The slow scanning direction is indicated by orange arrows. The images marked by $\Sigma_{1}$ to $\Sigma_{3}$ are the corrected images $\Psi_{1}$ to $\Psi_{3}$ from Fig. 8. As up and down images are congruent, we can use these images as a validation of our drift compensation routine.

$$
\dot{y}=(1.36 \pm 0.06) \mathrm{pm} / \mathrm{s} .
$$

We obtained virtually identical drift velocities from the corresponding up images (not shown).

To perform the lateral drift correction as described above, we use the open source software GWYDDION. The coordinates in the polynomial distortion tool are defined on the interval $[0,1]$. Thus, to use the polynoms presented in Eqs. (22)-(25), they have to be modified by the substitutions $H \rightarrow 1$ and $B$ $\rightarrow 1$. Furthermore, $\dot{x}$ and $\dot{y}$ have to be normalized to $[0,1]$. This is accomplished by the substitutions $\dot{x} \rightarrow \dot{x} / B$ and $\dot{y}$ $\rightarrow \dot{y} / H$.

Lateral drift correction with the polynomial distortion tool of GWYDDION is then carried out according to

$$
P_{x, \downarrow}^{\mathrm{Gwy}}(x, y)=x+\frac{-T \frac{\dot{x}}{B}}{1+T \frac{\dot{y}}{H}} y,
$$

$$
P_{y, \downarrow}^{\mathrm{Gwy}}(x, y)=\frac{1}{1+T \frac{\dot{y}}{H}} y,
$$

$P_{x, \uparrow}^{\mathrm{Gwy}}(x, y)=\frac{-T \frac{\dot{x}}{B}}{1-T \frac{\dot{y}}{H}}+x+\frac{T \frac{\dot{x}}{B}}{1-T \frac{\dot{y}}{H}} y$,

$$
P_{y, \uparrow}^{\mathrm{Gwy}}(x, y)=\frac{-T \frac{\dot{y}}{H}}{1-T \frac{\dot{y}}{H}}+\frac{1}{1-T \frac{\dot{y}}{H}} y .
$$

Using these polynomials, the drift velocities [Eq. (26) and (27)], the image dimensions $H=B=9.8 \mathrm{~nm}$, and the total 
image time $T=557 \mathrm{~s}$, we arrive at the merged images shown in Fig. 9. Each image is perfectly overlapping with the preceding and the consecutive image.

In this case of an unknown surface structure, the only criterion to verify the correctness of a drift correction strategy is comparing a corrected up with a corrected down image. These images need to be congruent in the overlap region. As evident from Fig. 9, this criterion is nicely fulfilled for our images except for small deviations due to scanner creep. This clearly demonstrates the validity of the presented drift correction algorithm and justifies the assumption of linear drift.

\section{CONCLUSION}

We presented a straightforward recipe for measuring and correcting both vertical and lateral linear drifts in SPM images after data acquisition. Vertical drift velocity is corrected by a pixelwise plane-substraction procedure that leaves sample tilt unaffected. Using the presented algorithm for lateral drift correction requires consecutive images with a stationary feature. The presented method is based on tracking this stationary feature over several images to measure the drift velocities. We derive polynomial equations, which can be used to correct for the image distortion induced by linear drift. Ready-to-use formulas are presented that can directly be used in the open source software GWYDDION. Thus, the present article constitutes a "users guide to drift correction."

\section{ACKNOWLEDGMENT}

Financial support from the Deutsche Forschungsgemeinschaft (DFG) through the Emmy Noether grant KU 1980/1-2 is gratefully acknowledged.

${ }^{1}$ F. Besenbacher, Rep. Prog. Phys. 59, 1737 (1996).

${ }^{2}$ F. J. Giessibl, Rev. Mod. Phys. 75, 949 (2003).

${ }^{3}$ See: http://www.gwyddion.net.
${ }^{4}$ J. Wintterlin, J. Trost, S. Renisch, R. Schuster, T. Zambelli, and G. Ertl, Surf. Sci. 394, 159 (1997).

${ }^{5}$ O. M. Magnussen, W. Polewska, L. Zitzler, and R. J. Behm, Faraday Discuss. 121, 43 (2002).

${ }^{6}$ M. J. Rost et al., Rev. Sci. Instrum. 76, 053710 (2005).

${ }^{7}$ M. Abe, Y. Sugimoto, Ó. Custance, and S. Morita, Appl. Phys. Lett. 87, 173503 (2005).

${ }^{8}$ M. Abe, Y. Sugimoto, Ó. Custance, and S. Morita, Nanotechnology 16, 3029 (2005).

${ }^{9}$ M. Abe, Y. Sugimoto, T. Namikawa, K. Morita, N. Oyabu, and S. Morita, Appl. Phys. Lett. 90, 203103 (2007).

${ }^{10}$ D. W. Pohl and R. Möller, Rev. Sci. Instrum. 59, 840 (1988).

${ }^{11}$ P. Rerkkumsup, M. Aketagawa, K. Takada, Y. Togawa, N. T. Thinh, and Y. Kozuma, Rev. Sci. Instrum. 75, 1061 (2004).

${ }^{12}$ B. S. Swartzentruber, Phys. Rev. Lett. 76, 459 (1996).

${ }^{13}$ M. Krueger, B. Borovsky, and E. Ganz, Surf. Sci. 385, 146 (1997).

${ }^{14}$ Y. Sugimoto, P. Pou, M. Abe, P. Jelínek, R. Pérez, S. Morita, and Ó. Custance, Nature (London) 446, 64 (2007).

${ }^{15}$ Y. Sugimoto, P. Pou, Ó. Custance, P. Jelínek, M. Abe, R. Pérez, and S. Morita, Science 322, 413 (2008).

${ }^{16}$ J. F. Jørgensen, L. L. Madsen, J. Garnaes, K. Carneiro, and K. Schaumburg, J. Vac. Sci. Technol. B 12, 1698 (1994).

${ }^{17}$ K. Henriksen and S. L. S. Stipp, Am. Mineral. 87, 5 (2002).

${ }^{18}$ R. Staub, D. Alliata, and C. Nicolini, Rev. Sci. Instrum. 66, 2513 (1995).

${ }^{19}$ B. A. Mantooth, Z. J. Donhauser, K. F. Kelly, and P. S. Weiss, Rev. Sci. Instrum. 73, 313 (2002).

${ }^{20}$ O. Sollbohmer, K. P. May, and M. Anders, Thin Solid Films 264, 176 (1995).

${ }^{21}$ V. Y. Yurov and A. N. Klimov, Rev. Sci. Instrum. 65, 1551 (1994).

${ }^{22}$ R. V. Lapshin, Meas. Sci. Technol. 18, 907 (2007).

${ }^{23}$ J. E. T. Andersen and P. Møller, Surf. Coat. Technol. 67, 213 (1994).

${ }^{24}$ J. T. Woodward and D. K. Schwartz, J. Vac. Sci. Technol. B 16, 51 (1998).

${ }^{25}$ Q. Yang, S. Jagannathan, and E. W. Boharman, IEEE Trans. Nanotechnol. 7, 209 (2008).

${ }^{26}$ See: http://www.gwyddion.net/documentation/user-guide/.

${ }^{27}$ W. H. Huang, W. W. Wang, A. D. Xia, N. Jin, and Z. Q. Hu, J. Vac. Sci. Technol. B 18, 2027 (2000).

${ }^{28}$ L. G. Brown, ACM Comput. Surv. 24, 325 (1992).

${ }^{29} \mathrm{P}$. Thévenaz, U. E. Ruttimann, and M. Unser, IEEE Trans. Image Process. 7, 27 (1998).

${ }^{30}$ See: http://gxsm.sourceforge.net/.

${ }^{31}$ R. Bechstein, M. Kitta, J. Schütte, A. Kühnle, and H. Onishi, J. Phys. Chem. C 113, 13199 (2009). 